\title{
Multiplexed Ultrasensitive Determination of Adrenocorticotropin and Cortisol Hormones at a Dual Electrochemical Immunosensor
}

\author{
María Moreno-Guzmán, Araceli González-Cortés, Paloma Yáñez-Sedeño, ${ }^{*}$ José M. Pingarrón \\ Department of Analytical Chemistry, Faculty of Chemistry, University Complutense of Madrid, Ciudad Universitaria s/n, \\ 28040-Madrid, Spain \\ *e-mail: yseo@quim.ucm.es
}

Received: February 2, 2012

Accepted: March 7, 2012

\begin{abstract}
A novel dual electrochemical immunosensor for the multiplexed determination of adrenocorticotropin (ACTH) and cortisol is reported. Aminophenylboronic acid-modified dual screen-printed carbon electrodes were prepared on which the corresponding ACTH and cortisol antibodies were immobilized. Competitive immunoassays involved biotinylated ACTH and alkaline phosphatase labelled streptavidin, or alkaline phosphatase labelled cortisol. Differential pulse voltammetry upon 1-naphtyl phosphate addition was employed to monitor the affinity reactions. The ranges of linearity were $5.0 \times 10^{-5}-0.1$ and $0.1-500 \mathrm{ng} / \mathrm{mL}$ for ACTH and cortisol. The usefulness of the dual immunosensor was demonstrated by analyzing certified human serum samples with good recoveries.
\end{abstract}

Keywords: Adrenocorticotropin, Cortisol, Electrochemical immunosensor, Phenyl boronic acid

DOI: 10.1002/elan.201200070

\section{Introduction}

Cortisol (hydrocortisone) is a glucocorticoid produced by the adrenal gland in response to stress and a low level of glucocorticoids in blood. Glucocorticoids play an essential role in the catabolism of peripheral fat, carbohydrates and proteins. In addition, cortisol has anti-inflammatory and immune modulatory effects. The secretion of cortisol is related to that of adrenocorticotropin hormone $(\mathrm{ACTH})$ through corticotropin-releasing hormone $(\mathrm{CRH}) . \mathrm{CRH}$ is synthesized and secreted by the hypotalamus. It stimulates pituitary corticotrophic cells to produce ACTH, a 39-amino acid peptide, which is a fragment of the larger molecule propiomelanocortintin (POMC). ACTH promotes growth and hormone synthesis by the adrenal cortex, and stimulates secretion of glucocorticoids, mineralocorticoids and androgenic steroids [1]. As indicated in the scheme depicted in Figure 1 [2], the amount of cortisol secreted by the adrenal gland produces an inhibitory effect on both the synthesis and secretion of $\mathrm{CRH}$ and ACTH through a negative feedback loop to the hypothalamus and pituitary glands, respectively. Serum levels of ACTH fluctuate with larger peaks in the early morning and nadiring in the late afternoon or early evening, which results in a parallel diurnal rhythm of cortisol secretion, with peak serum levels in the morning and lower levels in the evening. Stress, pain, trauma, hypoxia, hypothermia and hypoglycemia can increase the serum levels of ACTH. Perturbations of the hypothalamus-pituitary adrenal axis result in different clinical forms of hypercorticosolism, including Cushing's disease (pituitary adenoma), ectopic ACTH, ectopic CRH, and primary adrenal causes [3]. The diagnosis of Cushing's syndrome remains nowadays as one of the most challenging problems in clinical endocrinology [4]. Among other causes, this is because clinical manifestations may overlap with many common clinical conditions. For example, studies have shown that $2-3 \%$ of patients with poorly controlled type 2 diabetes mellitus have unrecognised Cushing's syndrome [5]. This diagnosis requires biochemical confirmation of excess cortisol excretion and ACTH determination in order to classify patients with endogenous hypercortisolemia into ACTH independent and dependent [6]. Different levels of ACTH and cortisol concentrations can be found in serum or urine depending on the type of disease. Typically, ACTH is decreased in adrenal-dependent Cushing's syndrome and maintained/elevated in Cushing's disease [7]. As an example, ACTH levels are undetectable or low $(<5 \mathrm{pg} / \mathrm{mL})$ and cortisol levels are high $(>15 \mu \mathrm{g} /$ $\mathrm{dL})$ in patients with primary adrenal tumours or hyperplasia, whereas patients with corticotropin-dependent Cushing's syndrome have high plasma ACTH levels $(>15$ $\mathrm{pg} / \mathrm{mL}$ ). Therefore, the development of analytical methods able to provide multiplexed determination of ACTH and cortisol hormones should help to perform a faster classification of the patients as well as an easier diagnosis of this kind of diseases. Nevertheless, these methodologies require to be sufficiently sensitive, robust and selective [8].

Radioimmunoassays and immunochemiluminiscence assays are the most often used methodologies for clinical determination of cortisol and ACTH independently [4,7]. 


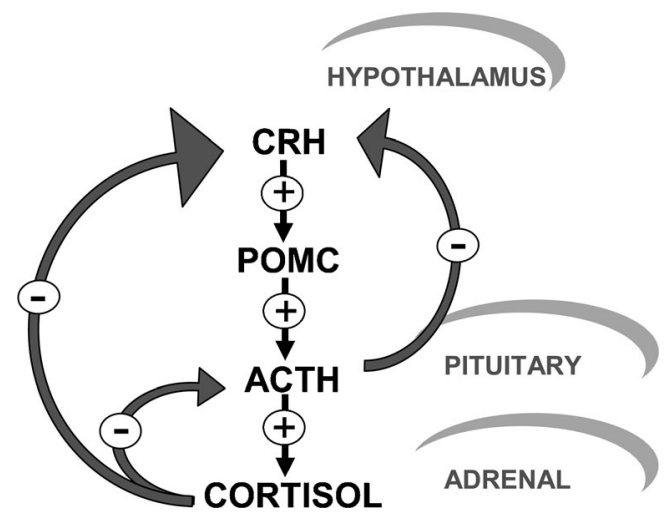

Fig. 1. Diagram illustrating the hypothalamus, pituitary and adrenal axis, and the stimulating or negative feedback from CRH, POMC, ACTH and cortisol. Modified from [2].

However, in some cases, the assays are not sensitive enough to determine the low concentrations of ACTH involved in ACTH-independent Cushing's syndrome [7].

Because of their high sensitivity, rapid response and usability, electrochemical immunosensors have demonstrated to be excellent alternatives in clinical analysis of selected analytes $[9,10]$. Our group has developed recently electrochemical immunosensors for the determination of some hormones. For example, testosterone was determined in serum using configurations based on the immobilization of anti-testosterone antibodies on gold nanoparticles-carbon nanotubes-Teflon composite electrodes [11]. Magnetic electrochemical immunosensors were also developed for testosterone [12], prolactin [13] and cortisol [14]. Moreover, the ultrasensitive detection of ACTH was accomplished by using disposable phenylboronic-modified electrochemical immunosensors [15].

In this paper, we report the preparation of a dual electrochemical immunosensor for the multiplexed determination of $\mathrm{ACTH}$ and cortisol, which satisfies the requirements of sensitivity, selectivity and reproducibility needed for clinical applications. Aminophenylboronic acid-modified electrodes were prepared on which the corresponding ACTH and cortisol antibodies were immobilized. Competitive immunoassays were then performed and differential pulse voltammetry upon 1-naphtyl phosphate addition was employed to monitor the affinity reactions. The usefulness of the approach was demonstrated by its application to serum samples.

\section{Experimental}

\subsection{Apparatus and Electrodes}

Electrochemical measurements were carried out using an 1030 B Electrochemical Analyzer from $\mathrm{CH}$ Instruments. Dual screen-printed electrodes (C 1110 DropSens, Oviedo, Spain) consisted of two elliptic carbon working electrodes, a carbon counter electrode and a silver pseudo-reference electrode. All experiments were per- formed at room temperature. A P-Selecta ultrasonic bath, and a P-Selecta Agimatic magnetic stirrer, all distributed by Scharlab, Madrid, Spain, were also used.

\subsection{Reagents and Solutions}

Anti-ACTH C-terminal was purchased from Fitzgerald MA, USA. $1.05 \mathrm{mg} / \mathrm{mL}$ solutions were prepared in phosphate buffer solution of pH 7.4 (PBS). Diluted solutions of both ACTH (Genway Biotech, CA, USA) and biotinylated ACTH (Biotin-ACTH) (AnaSpec, CA, USA) were prepared in PBS. Murine monoclonal anti-cortisol was from East Coast Bio, ME, USA. $2.39 \mathrm{mg} / \mathrm{mL}$ solutions were prepared in PBS. Cortisol labelled with alkaline phosphatase in position 3 (cortisol-AP), with an enzyme activity of $1840 \mathrm{U} / \mathrm{mg}$, was obtained from Cal Bioreagents, CA, USA. Diluted solutions were prepared daily in Tris buffer solution of $\mathrm{pH} 7.2$ which also contained $2 \%$ BSA. A $500 \mu \mathrm{g} / \mathrm{mL}$ stock solution of unlabelled cortisol (Aldrich $>98 \%$ ) in methanol was prepared. More diluted solutions were prepared in Tris buffer solution of $\mathrm{pH}$ 7.2. A $50 \mathrm{mM}$ Tris (Scharlau, 99\%) and $20 \mathrm{mM} \mathrm{NaCl}$ (Scharlau, $99 \%$ ) buffer solution of $\mathrm{pH} 7.2$, and a $0.1 \mathrm{M}$ Trizma base (Sigma, 99\%) and $1 \mathrm{mM}$ magnesium chloride (Panreac, $99 \%$ ) buffer solution of $\mathrm{pH} 9.0$, were employed. 1Naphthyl phosphate monosodium salt monohydrate (1NPP, Sigma) was also used. 0.05 M 1-NPP solutions were prepared daily in Trizma base buffer of pH 9.0. BSA (Gerbu, type $\mathrm{VH}$ ) was also used. 2\% BSA solutions in both PBS and Tris buffer solution of $\mathrm{pH} 7.2$ were used. Streptavidin labelled with alkaline phosphatase (StreptAP) (Sigma) was also employed. Diluted solutions were prepared in Tris buffer solution of $\mathrm{pH}$ 7.2. 4-Aminobenzoic acid (ABA, Across), 4-amino phenyl boronic acid (APBA, Sigma), 1-ethyl-3-[3-dimethylaminopropyl] carbodiimide hydro-chloride (EDC) (Sigma) and $N$-hydroxysuccinimide (NHS) (Sigma) were also used.

\subsection{Samples}

Samples containing different ACTH and cortisol mixtures were analyzed. These were prepared from certified lyophilised human sera containing $10 \mu \mathrm{g} / \mathrm{mL}$ ACTH (NIBSC, 74/555) or $277 \mathrm{ng} / \mathrm{mL}$ cortisol (European Reference Materials, ERM-DA 193, Sigma), in the reconstituted serum, respectively.

\subsection{Procedures}

\subsubsection{Modification of Dual SPC Electrodes}

Figure 2 a displays a scheme of the employed procedure. Firstly, $20 \mathrm{mg}$ of 4-aminobenzoic acid were dissolved in $2 \mathrm{~mL} 1 \mathrm{M} \mathrm{HCl}$ and cooled with ice. Then, the corresponding diazonium salt was prepared by adding dropwise a $2 \mathrm{mM}$ NaNO 2 aqueous solution $(38 \mu \mathrm{L}$ for each $200 \mu \mathrm{L}$ of 4-aminobenzoic acid solution) with constant stirring. Next, $40 \mu \mathrm{L}$ of the resulting solution were spotted onto 

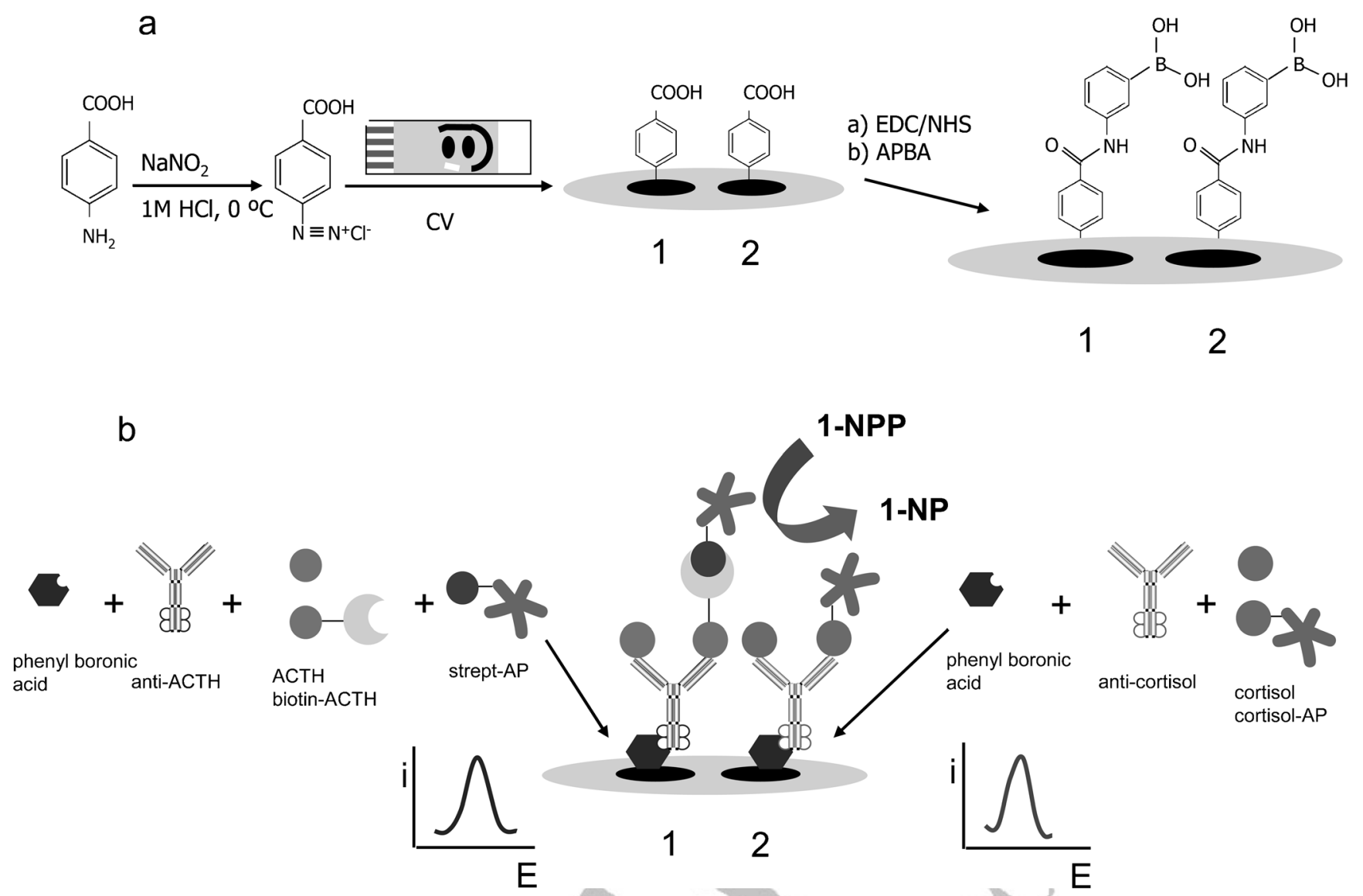

Fig. 2. Schematic picture for the preparation of the dual electrochemical immunosensor for multiplexed determination of ACTH and cortisol.

the SPC electrodes and ten successive voltammetric cycles from 0.0 to $-1.0 \mathrm{~V}(v=200 \mathrm{mV} / \mathrm{s})$ were scanned. Finally, the modified electrodes were washed thoroughly with water and methanol, and dried at room temperature. In a second step, $10 \mu \mathrm{L}$ of an EDC/NHS mixture $(0.1 \mathrm{M}$ each) aqueous solution were spotted onto the modified electrodes and left to react for $1 \mathrm{~h}$. After rinsing with water and methanol and allowing drying, $10 \mu \mathrm{L}$ of a $50 \mathrm{mM}$ 3-aminophenylboronic acid were placed onto the electrodes. After $3 \mathrm{~h}$ incubation, the resulting aminophenylboronic acid-modified electrodes (APBA/SPCEs) were thoroughly rinsed with water and methanol and allowing drying at room temperature.

\subsubsection{Preparation of the Immunosensors}

ACTH and cortisol immunosensors were prepared on APBA/SPCE1 and APBA/SPCE2 modified electrodes, respectively. $4 \mu \mathrm{L}$ of $30 \mu \mathrm{g} / \mathrm{mL}$ anti-ACTH and $50 \mu \mathrm{g} / \mathrm{mL}$ anti-cortisol solutions in PBS were dropped onto the respective electrode and left stand overnight at $8^{\circ} \mathrm{C}$. Then, $4 \mu \mathrm{L}$ of a $2 \%$ BSA blocking solution were deposited onto both electrodes and incubation was allowed for $1 \mathrm{~h}$ at room temperature. Competitive immunoassays were performed by spotting $4 \mu \mathrm{L}$ of a mixture solution of ACTH (or the sample) and $1 \mu \mathrm{g} / \mathrm{mL}$ biotinylated-ACTH (biotin-
ACTH) on the anti-ACTH/APBA/SPCE1 electrode, and $4 \mu \mathrm{L}$ of a mixture solution of cortisol (or the sample) and $1 \mu \mathrm{g} / \mathrm{mL}$ alkaline phosphatase-labelled cortisol 2\% BSA (AP-cortisol) on the anti-cortisol/APBA/SPCE2 electrode allowing incubation at both electrodes for $45 \mathrm{~min}$ at $8^{\circ} \mathrm{C}$. Thereafter, $4 \mu \mathrm{L}$ of $2 \mu \mathrm{g} / \mathrm{mL}$ alkaline phosphatase-labelled streptavidin (Strept-AP) and $4 \mu \mathrm{L}$ of Tris buffer solution of $\mathrm{pH} 7.2$ were added to the Biotin-ACTH/anti-ACTH/ APBA/SPCE1 and the cortisol-AP/anti-cortisol/APBA/ SPCE2, respectively. After $60 \mathrm{~min}$ incubation at $8{ }^{\circ} \mathrm{C}$, $45 \mu \mathrm{L}$ Trizma buffer plus $5 \mu \mathrm{L}$ of $0.05 \mathrm{M} 1$-NPP solution were deposited onto the electrodes and allowed to stand for 8 minutes. Differential pulse voltammograms were recorded over the -0.15 to $+0.60 \mathrm{~V}$ range to obtain the electroanalytical signals.

\subsubsection{Multiplexed Determination of ACTH and Cortisol in Serum}

Lyophilised human sera containing ACTH or cortisol were reconstituted, respectively, in $2.5 \mathrm{~mL}$ or $1.25 \mathrm{~mL}$ Milli-Q water to obtain $10 \mu \mathrm{g} / \mathrm{mL}$ ACTH, or $277 \mathrm{ng} / \mathrm{mL}$ cortisol stock solutions. From these stock solutions, different ACTH-cortisol mixtures also containing BiotinACTH or cortisol-AP conjugates were prepared and spotted on the anti-ACTH/APBA/SPCE1 anti-cortisol/ 
SPCE 1

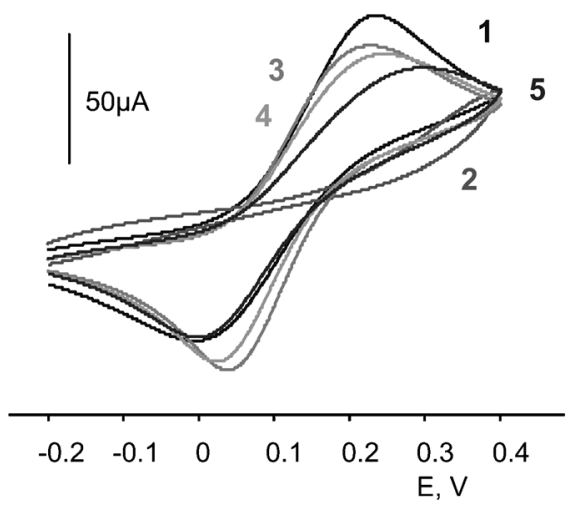

SPCE 2

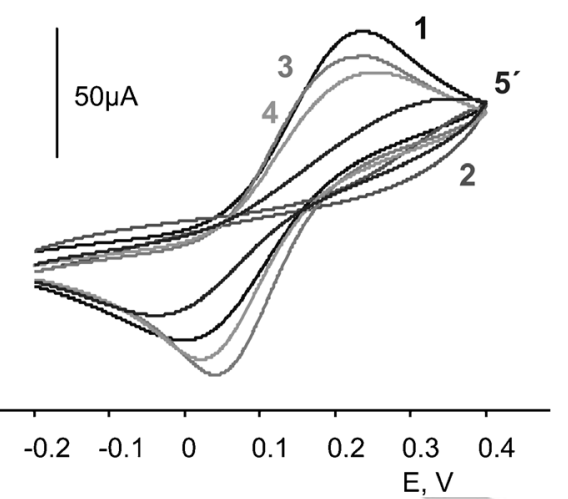

Fig. 3. Cyclic voltammograms recorded for a $5 \mathrm{mM}\left[\mathrm{Fe}(\mathrm{CN})_{6}\right]^{3-/ 4-} 0.1 \mathrm{M} \mathrm{KCl}$ solution at (1) bare SPCE; (2) ABA/SPCE; (3) activated ABA/SPCE; (4) APBA/SPCE; (5) anti-ACTH/APBA/SPCE; (5') anti-cortisol/APBA/SPCE; $v=100 \mathrm{mV} / \mathrm{s}$.

APBA/SPCE2 electrodes. Thereafter, the ACTH and cortisol were simultaneously determined by applying the procedure described above which involved simultaneous measurement of the respective DPV peak current values at each electrode and interpolation into the linear portion of the corresponding calibration plot obtained with ACTH or cortisol standard solutions.

\section{Results and Discussion}

The methodologies employed in the design and functioning of the dual immunosensor are illustrated in Figure 2. As it is described in Section 2.4.1, firstly, SPCE1 and SPCE2 were modified with boronic acid as described in the Experimental section (Procedure 2.4.1.). Subsequently, anti-ACTH and anti-cortisol antibodies were attached to the resulting APBA/SPCEs through the oriented boronic acid-saccharide bonds [16]. After a blocking step with BSA, competitive assays between ACTH and Biotin-ACTH, and between cortisol and cortisol-AP for the binding sites of the respective antibodies were accomplished. In the case of the ACTH immunosensor a further conjugation with streptavidin labelled with alkaline phosphatase was carried out. In both cases, 1-NPP was employed as AP substrate to detect the immunosensing events and the electrochemical oxidation of the generated enzyme reaction product, 1-naphtol, was measured by differential pulse voltammetry. Cyclic voltammetry from a $5 \mathrm{mM}[\mathrm{Fe}(\mathrm{CN}) 6]^{3-} /^{4-} 0.1 \mathrm{M} \mathrm{KCl}$ solution was employed to characterize the modified electrode surface of SPCE1 and SPCE2 upon the different modification steps (Figure 3). As expected, voltammograms 1 to 4 were similar at both electrodes.

Modification of SPCEs with ABA produced a sharp decrease in the voltammetric response due to the electrostatic repulsion between the redox probe and the negatively charged carboxylate groups. Upon activation of the surface-confined carboxyl groups with EDC/NHS and attachment of APBA moieties to the electrode surface through the formation of amide bonds (voltammograms 3 and 4), an increase in the voltammetric response to the redox probe was observed as a consequence of the electrode negative surface charge neutralization. Conversely, the peak current measured at the anti-ACTH/APBA/ SPCE1 (curve 5) was remarkably higher than that obtained with the anti-cortisol/APBA/SPCE2 (curve 5'), thus reflecting differences in the antibodies loadings and characteristics.

\subsection{Optimization of the Experimental Variables Involved in the Immunosensors Responses}

All variables involved in the electroanalytical responses of the dual immunosensor for ACTH and cortisol were optimized. Firstly, the respective antibody loadings into the APBA/SPCE1 and APBA/SPCE2 were evaluated by checking the DP voltammetric responses obtained with different immunosensors prepared with no ACTH or cortisol and by spotting $4 \mu \mathrm{L}$ of anti-ACTH or anti-cortisol solutions prepared in the 0 to $80 \mu \mathrm{g} / \mathrm{mL}$ antibody concentration range, respectively. $1 \mu \mathrm{g} / \mathrm{mL}$ Biotin-ACTH M 1NPP were used in these experiments. Figure 4a shows as the measured $i_{\mathrm{p}}$ values increased both at the ACTH and cortisol immunosensors with the antibody loading, exhibiting a remarkably lower increase from $30 \mu \mathrm{g} / \mathrm{mL}$ antiACTH and $50 \mu \mathrm{g} / \mathrm{mL}$ anti-cortisol. Therefore, these antibodies loading values were selected for further work. Furthermore, anti-ACTH/APBA/SPCE1 and anti-cortisol/ APBA/SPCE2 were allowed to stay overnight at $8^{\circ} \mathrm{C}$.

In order to reduce non-specific binding of immunoreagents on the electrodes surfaces, a blocking step with BSA was accomplished. In a first approach, the experimental conditions optimized previously for ACTH (2\% BSA and $1 \mathrm{~h}$ incubation time) [15] were used. Although the unspecific responses from Strept-AP at SPCE were found to be always lower than $10 \%$ of the signals obtained with the Strept-AP/Biotin-ACTH/anti-ACTH/ APBA/SPCE, the nonspecific binding was remarkably higher when cortisol-AP was involved. Figure 5 displays

\footnotetext{
40 www.electroanalysis.wiley-vch.de र

Electroanalysis 2012, 24, No. $\square, 1-9$ 


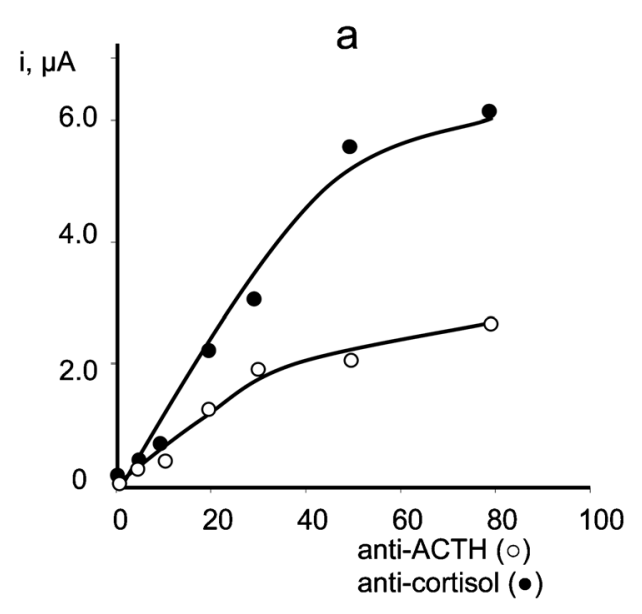

C

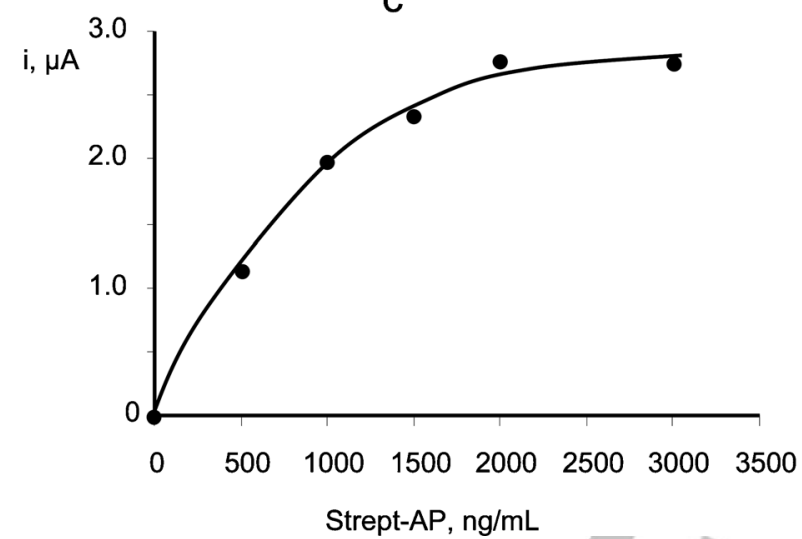

b

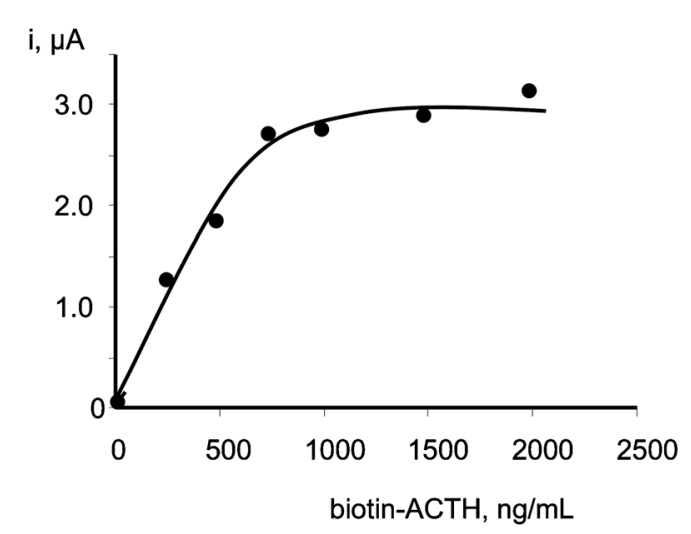

d

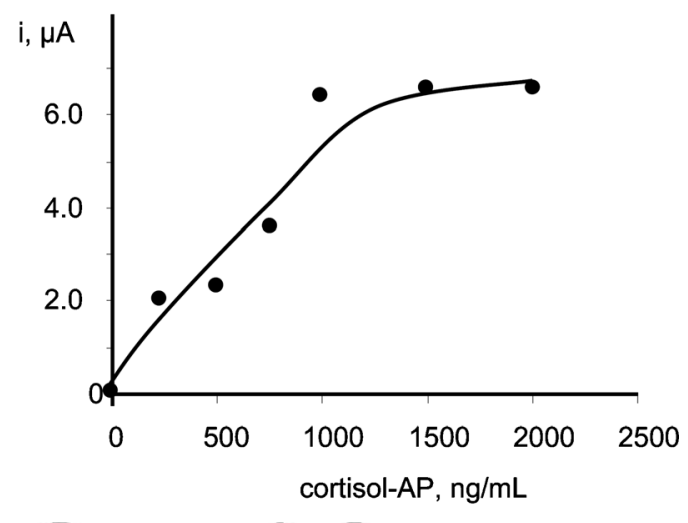

Fig. 4. Optimization of the different experimental variables involved in the preparation of the dual immunosensor for ACTH and cortisol.

DP voltammograms recorded at SPCEs from cortisol-AP solutions without blocking with BSA (curve 1) and with a blocking step with $2 \%$ BSA for different incubation times (curves 2 and 3). The DP voltammograms recorded upon spotting a cortisol-AP-2\% BSA mixture solution with and without a further incubation step with $2 \%$ BSA are also displayed (curves 4 and 5). As it can be observed, the lower unspecific signal was obtained when the cortisol-AP solution was prepared in the presence of $2 \%$ BSA, and the electrode was also blocked with $2 \%$ BSA during $1 \mathrm{~h}$ incubation time. Using these experimental conditions, Figure $5 \mathrm{~b}$ compares the differential pulse voltammograms obtained in the measurement of the specific response of the cortisol-AP/anti-cortisol/APBA/SPCE immunosensor for $0 \mathrm{ng} / \mathrm{mL}$ cortisol (curve 1) and $250 \mathrm{ng} / \mathrm{mL}$ cortisol (curve 2), with the unspecific voltammetric signal (curve 3). As it can be seen, a large analytical signal-tobackground current ration could be obtained thus allowing the sensitive determination of cortisol to be performed with the dual immunosensor.

The concentration of Biotin-ACTH was optimized by preparing various Strept-AP/Biotin-ACTH/anti-ACTH/ APBA/SPCE immunosensors which were incubated in Biotin-ACTH solutions with different concentrations ranging between 0 and $2 \mu \mathrm{g} / \mathrm{mL}$. Figure $4 \mathrm{~b}$ shows as the DP peak current measured for $0 \mathrm{ng} / \mathrm{mL}$ ACTH exhibited a rapid increase from 0 to $0.75 \mu \mathrm{g} / \mathrm{mL}$ and levelled off for higher Biotin-ACTH concentrations. Accordingly, $1 \mu \mathrm{g} /$ $\mathrm{mL}$ Biotin-ACTH concentration was selected for further work. Moreover, $45 \mathrm{~min}$ at $8^{\circ} \mathrm{C}$ was the optimized value for incubation of the anti-ACTH/APBA/SPCE immunosensors with the Biotin-ACTH solution (results not shown).

As it was illustrated in Figure 1, Strept-AP was used as the labelled protein in the immunosensor design to determine ACTH. The conjugate concentration was optimized by testing the voltammetric response of different immunosensors incubated with Strept-AP in the 0 to $3 \mu \mathrm{g} / \mathrm{mL}$ range. The obtained results (Figure $4 \mathrm{c}$ ) revealed a rapid current increase up to $2 \mu \mathrm{g} / \mathrm{mL}$ Strept-AP and a practically constant value for larger conjugate concentrations. Therefore, that concentration, which probably corresponded to the saturation of the biotinylated ACTH, was selected for further work. Moreover, optimal results were achieved when the incubation step with the Strept-AP solution was performed at $8^{\circ} \mathrm{C}$ for 60 minutes (results not shown).

Similarly, the cortisol-AP concentration was optimized in the case of the cortisol immunosensor preparation. 
a)

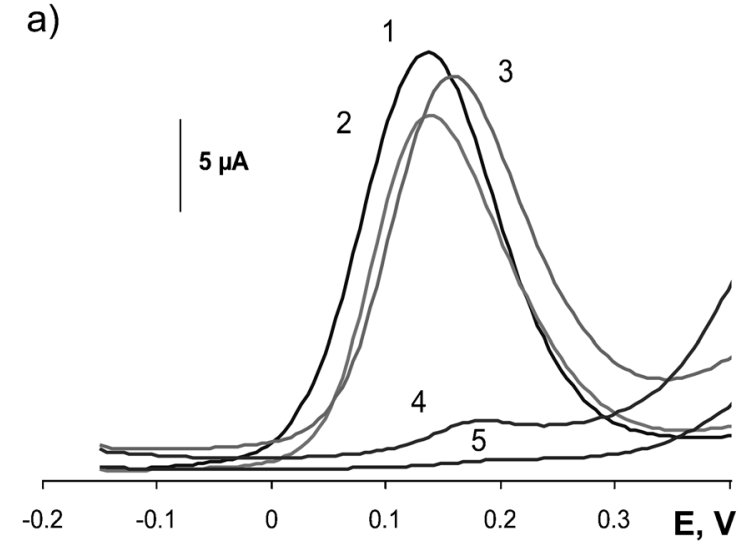

b)

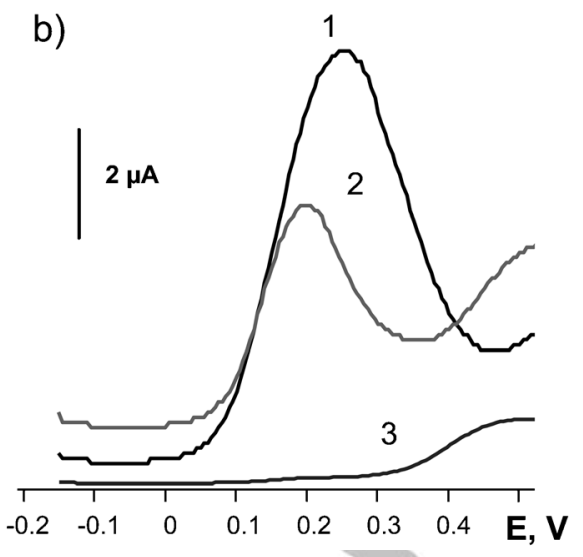

Fig. 5. a) Unspecific DPV responses from $1 \mu \mathrm{g} / \mathrm{mL}$ cortisol-AP at SPCEs (1); $1 \mu \mathrm{g} / \mathrm{mL}$ cortisol-AP and $1 \mathrm{~h}$ incubation in $2 \%$ BSA (2) $; 1 \mu \mathrm{g} / \mathrm{mL}$ cortisol-AP and $2 \mathrm{~h}$ incubation in $2 \%$ BSA (3); $1 \mu \mathrm{g} / \mathrm{mL}$ cortisol-AP $+2 \%$ BSA (4); $1 \mu \mathrm{g} / \mathrm{mL}$ cortisol-AP $+2 \%$ BSA and $1 \mathrm{~h}$ incubation in $2 \%$ BSA (5); $0.05 \mathrm{M}$ 1-NPP; b) Specific (1), competitive (2) and unspecific DPV responses obtained with an anticortisol/APBA/SPCE immunosensor for $0 \mathrm{ng} / \mathrm{mL}$ (1) and $250 \mathrm{ng} / \mathrm{mL}$ (2) cortisol; the unspecific response (3) was measured as in a) (5). $50 \mu \mathrm{g} / \mathrm{mL}$ anti-cortisol; $1 \mu \mathrm{g} / \mathrm{mL}$ cortisol-AP; $0.05 \mathrm{M}$ 1-NPP.

This was made by checking the DP voltammetric response of various immunosensors incubated with the conjugate in the 0 to $2 \mu \mathrm{g} / \mathrm{mL}$ concentration range. Figure $4 \mathrm{~d}$ shows a rapid increase in the ip value with the conjugate concentration at the lower cortisol-AP concentration values reaching a constant response from $1 \mu \mathrm{g} / \mathrm{L}$, indicating saturation of the antibodies binding sites. Using this conjugate concentration, an incubation time of $45 \mathrm{~min}$ at $8^{\circ} \mathrm{C}$ resulted appropriate to allow an adequate competition between cortisol and cortisol-AP for the binding positions of anti-cortisol.

As it was mentioned already, 1-NPP was the alkaline phosphatase substrate employed to generate the enzymatic product suitable to be voltammetrically detected at the modified electrodes surface for both immunosensors [14]. Thus, the influence of 1-NPP concentration on the simultaneous differential pulse voltammetric responses of both the Strept-AP/Biotin-ACTH/anti-ACTH/APBA/SPCE1 and cortisol-AP/anti-cortisol/APBA/SPCE2 immunosensors was evaluated in the $5 \times 10^{-6}$ to $0.1 \mathrm{M}$ range (results not shown). The highest peak current values were achieved using a $50 \mathrm{mM} 1$-NPP concentration, which represented a relatively high value suitable to ensure that the enzyme reaction rate depended only on the enzyme concentration. Finally, $8 \mathrm{~min}$ was selected as an optimal hydrolysis time of the enzyme substrate. The possible crosstalk between the two SPCEs and reaction products was evaluated by recording DP yoltammograms at the immunosensors where mixture solutions of ACTH and cortisol were deposited. Figure 6 compares the voltammograms recorded from $50 \mathrm{ng} / \mathrm{mL}$ ACTH plus $1 \mu \mathrm{g} / \mathrm{mL}$ cortisol-AP (curve 1) and only $1 \mu \mathrm{g} / \mathrm{mL}$ cortisol-AP (curve 2) at the cortisol-AP/anti-cortisol/APBA/SPCE2. Similarly, voltammograms recorded with the Strept-AP/Biotin-ACTH/antiACTH/APBA/SPCE1 for both $500 \mathrm{ng} / \mathrm{mL}$ cortisol plus $1 \mu \mathrm{g} / \mathrm{mL}$ Biotin-ACTH (curve 3) and only $1 \mu \mathrm{g} / \mathrm{mL}$ Biotin-ACTH (curve 4) are also displayed in Figure 6. As it can be observed, no significant differences in the vol-

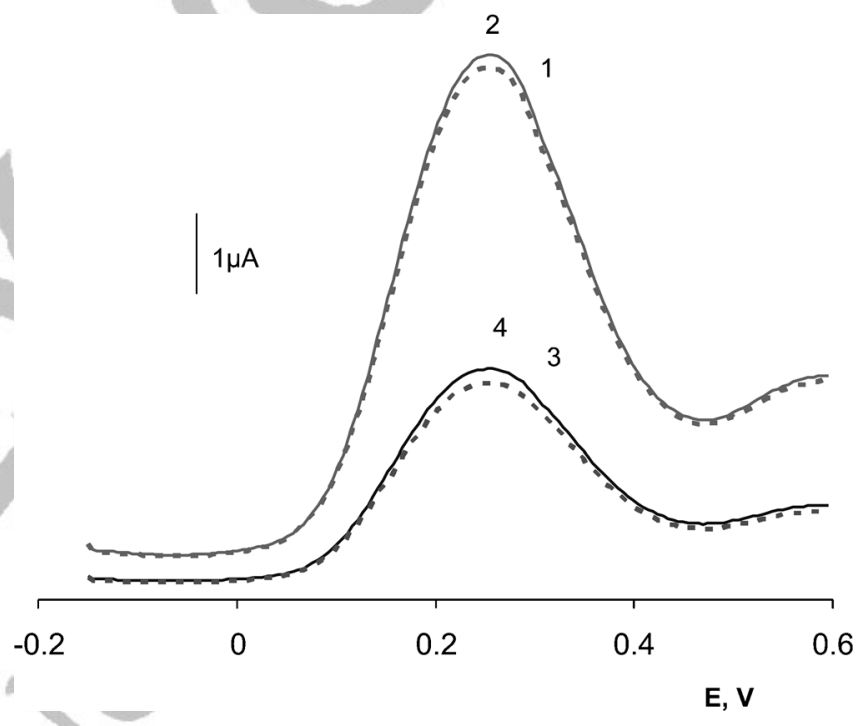

Fig. 6. DPVresponses from: cortisol-AP + ACTH/anti-cortisol/ APBA/SPCE2 (curve 1 -.-); cortisol-AP/anti-cortisol/APBA/ SPCE2 (curve $2-$ ); Strept-AP/Biotin-ACTH + cortisol/antiACTH/APBA/SPCE1 (curve 3 -.--); Strept-AP/Biotin-ACTH/ anti-ACTH/APBA/SPCE1 (curve $4-$ ).

tammetric responses with each immunosensor were found in the presence and in the absence of the non-target analyte, thus demonstrating that no appreciable cross-talking occurred with the developed dual immunosensor.

\subsection{Analytical Characteristics for ACTH and Cortisol Determination with the Dual Immunosensor}

Figure 7 shows the calibration plots for ACTH and cortisol obtained by differential pulse voltammetry under the optimized conditions stated above. The concentrations of ACTH and cortisol were measured between the $5 \times 10^{-8}$ 50 and $5 \times 10^{-4}-5 \times 10^{3} \mathrm{ng} / \mathrm{mL}$ ranges, respectively. The corresponding analytical characteristics are summarized 


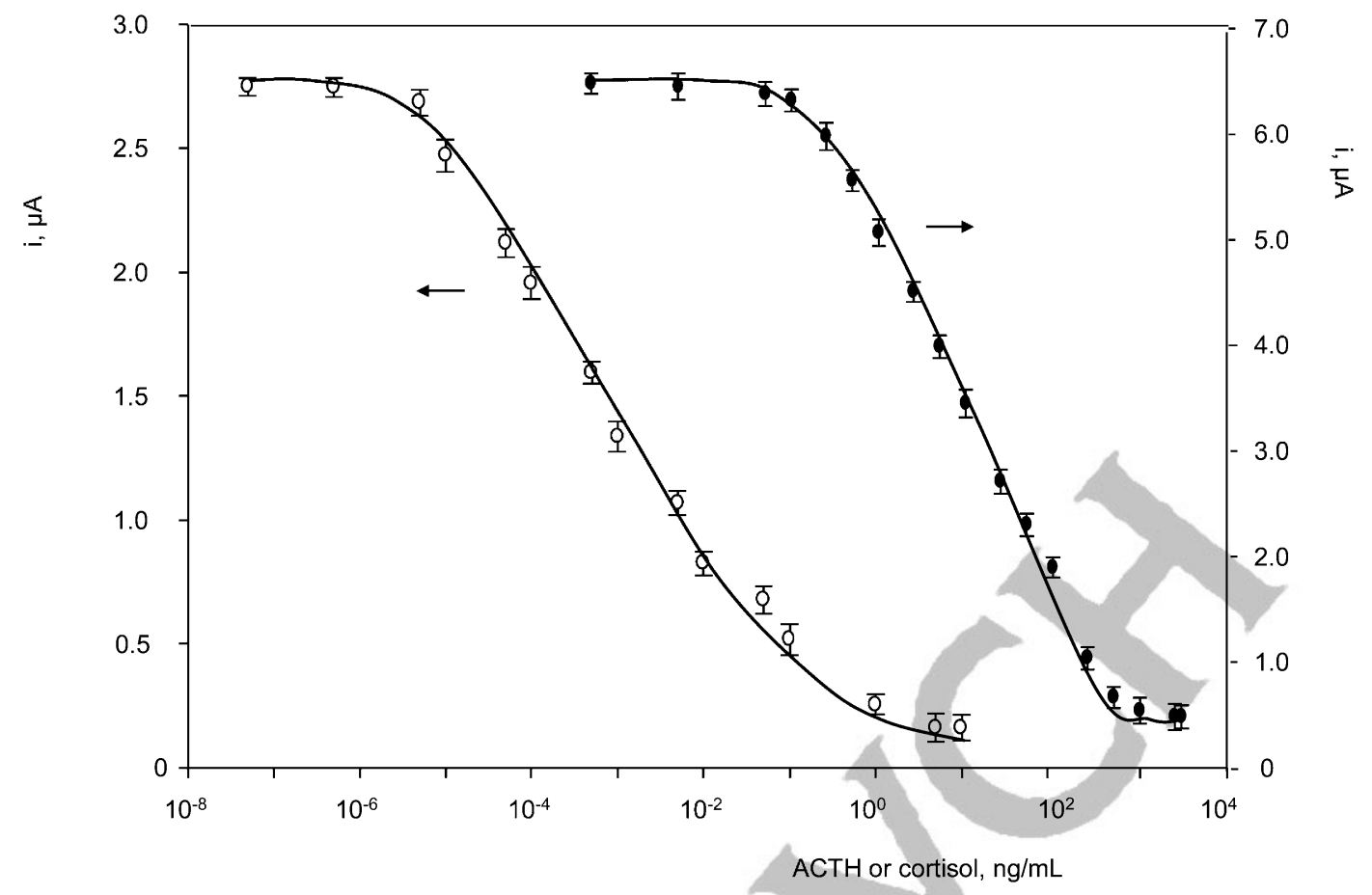

Fig. 7. Calibration plots for ACTH $(\circ)$ and cortisol $(\bullet)$ obtained with the Strept-AP/Biotin-ACTH/anti-ACTH/APBA/SPCE1 and cortisol-AP/anti-cortisol/APBA/SPCE2 immunosensors.

cortisol). This value was $\pm 0.24 \mu \mathrm{A}$ for $\mathrm{ACTH}$ and in Table 1 . The $i_{\mathrm{p}}$ vs. concentration curves were fitted by $\pm 0.12 \mu \mathrm{A}$ for cortisol, with relative standard deviations of non-linear regression using the Sigma Plot data analysis $6.3 \% \quad(n=5)$ for both compounds. Accordingly, $L O D$ software. The adjusted equation was:

$i_{p}=\frac{i_{\max }-i_{\min }}{1+E C_{50} / x^{\mathrm{h}}}+i_{\min }$

with $r=0.998(\mathrm{ACTH})$ and $r=0.999$ (cortisol), where $i_{\max }$ and $i_{\min }$ were the maximum and minimum current values of the calibration graphs: $2.92 \pm 0.07$ and $0.093 \pm$ $0.006 \mu \mathrm{A}$, respectively, for $\mathrm{ACTH}$, and $6.64 \pm 0.01$ and $0.038 \pm 0.008 \mu \mathrm{A}$, respectively, for cortisol. As can be seen in Table 1, both calibration graphs provided wide linear concentration ranges, this being in agreement with the low Hill slope values [17]. The limits of detection were calculated from the equation [18]:

$L O D=E C_{50}\left(\frac{i_{\max }-i_{\min }}{i_{\max }-i_{\min }-3 s}-1\right)^{-1 / h}$

where $s$ is the standard deviation $(n=10)$ of the zero value (the $i_{\mathrm{p}}$ value measured in the absence of ACTH or values of $40 \mathrm{pg} / \mathrm{L} \mathrm{ACTH}$ and $37 \mathrm{pg} / \mathrm{mL}$ cortisol were achieved. These analytical characteristics are much better than those reported previously in the literature for the individual determination of ACTH or cortisol using other approaches and similar to those reported recently by our group. In fact, various immunoassays were proposed for the determination of ACTH based on different antibodies and using radioactivity, chemiluminiscence or fluorescence as detection techniques. These methodologies allowed ranges between a few units up to various hundreds of ng/L ACTH to be covered [19-21], concentrations that are much larger than those appearing in the linear range obtained for this hormone with the developed Strept-AP/ Biotin-ACTH/anti-ACTH/APBA/SPCE1 immunosensor. On the other hand, the electrochemical immunosensor for ACTH previously described by our group provided a detection limit of $18 \mathrm{pg} / \mathrm{L}$ [15]. This slight difference is most likely due to the remarkably smaller surface area of the SPCE working electrodes in the dual device $\left(5.6 \mathrm{~mm}^{2}\right)$ with respect to that of the single SPCE used for the individual determination of ACTH $\left(12.56 \mathrm{~mm}^{2}\right)$.

Table 1. Analytical characteristics of the multiple electrochemical immunosensor for the simultaneous determination of ACTH and cortisol.

\begin{tabular}{llllll}
\hline Analyte & Linear range $(\mathrm{ng} / \mathrm{mL})$ & $r$ & $E C_{50}(\mathrm{ng} / \mathrm{mL})$ & Hill slope & $L O D(\mathrm{pg} / \mathrm{mL})$ \\
\hline ACTH & $5.0 \times 10^{-5}-0.1$ & 0.991 & $0.0007 \pm 0.0002$ & $-0.37 \pm 0.03$ & 0.04 \\
Cortisol & $0.1-500$ & 0.996 & $12 \pm 2$ & $-0.50 \pm 0.03$ & 37 \\
\hline
\end{tabular}




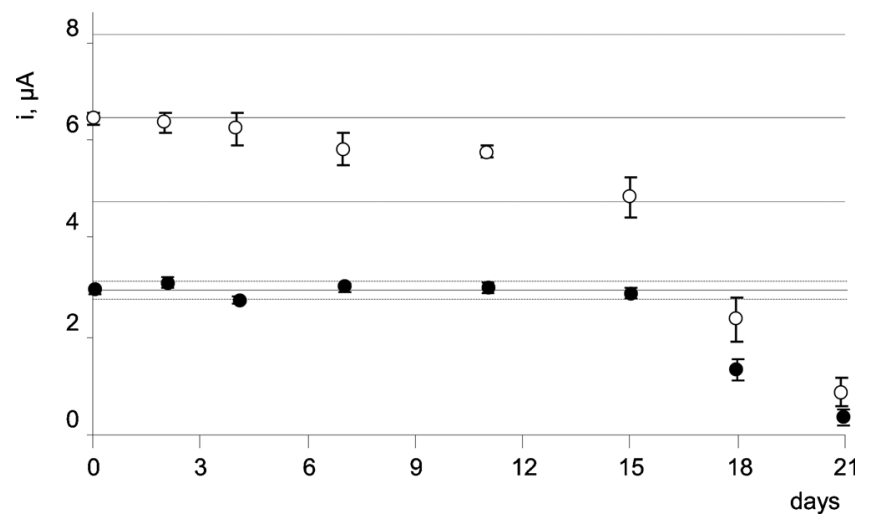

Fig. 8. Control chart constructed for anti-ACTH/APBA/SPCE1 $(\bullet)$ and anti-cortisol/APBA/SPCE $(\bigcirc)$. Each point corresponds to the mean value for three successive measurements of $5 \mathrm{mM} 1$ NPP.

Regarding cortisol, the limit of detection achieved in this work with the cortisol-AP/anti-cortisol/APBA/SPCE2 immunosensor was one half of the smallest reported value $(72 \mathrm{pg} / \mathrm{mL})$ obtained by a non-competitive colorimetric ELISA using cortisol-poly(L-lys) conjugates and HRPcortisol [22]. Comparing with a previous immunosensor reported by our group using SPCEs and protein-A functionalized magnetic microparticles [14], the limit of detection is approximately ten times higher but the range of linearity is wider. This difference is due not only to the smaller electrochemical surface area of SPCE2 compared with the conventional SPCEs used in [14], but also to the ability of magnetic microparticles to immobilize a high concentration of biomolecules onto the electrode surface allowing the achievement of high sensitivities.

It is important to remark that the limits of detection achieved for both analytes with the dual immunosensor fulfils the requirements for the analysis of the hormones in human serum considering that in typical diseases the ACTH and cortisol concentrations are around $0.005 \mathrm{ng} /$ $\mathrm{mL}$ and $150 \mathrm{ng} / \mathrm{mL}$, respectively.

The reproducibility of the DP voltammetric measurements was evaluated by carrying out repetitive assays in the same day and in different days for solutions containing no ACTH or cortisol and for $1 \mathrm{pg} / \mathrm{mL}$ ACTH or $10 \mathrm{ng} / \mathrm{mL}$ cortisol (concentrations similar to the respective EC50 values). Relative standard deviation (RSD) values of $6.3 \%(n=8)$ were obtained for the assays performed on the same day with both ACTH and cortisol immunosensors, whereas the RSD values $(n=8)$ were $7.6 \%$ when no ACTH was present and $8.5 \%$ for $1 \mathrm{pg} / \mathrm{mL}$ $\mathrm{ACTH}$, and $7.9 \%$ without cortisol and $8.1 \%$ for $10 \mathrm{ng} /$ $\mathrm{mL}$ cortisol, when the measurements were carried out on different days. The storage stability of the anti-ACTH/ APBA/SPCE1, Strept-AP/Biotin-ACTH/anti-ACTH/ APBA/SPCE1, anti-cortisol/APBA/SPCE2 and cortisolAP/anti-cortisol/APBA/SPCE2 immunosensors was also evaluated. In order to do that, different bioelectrodes were prepared on the same day and stored in a refrigerator at $4{ }^{\circ} \mathrm{C}$. Then, each bioelectrode was used to construct the corresponding immunosensor and to measure the voltammetric response for $5 \mathrm{mM} 1-\mathrm{NPP}$ in the absence of ACTH or cortisol. As an example, Figure 8 shows the control chart displaying the results obtained for a period of time of 21 days for anti - ACTH/APBA/SPCE1 (•) and anti-cortisol/APBA/SPCE2 $(\bigcirc)$ immunosensors. Each point in the control chart represented the mean value of three successive measurements and the control limits were set at \pm 3 times the standard deviation calculated for the whole series of experiments. As it can be seen, both bioelectrodes yielded voltammetric responses that remained inside the control limits for 15 days. On the other hand, the storage stability of the bioelectrodes constructed with all the bioconjugates, Strept-AP/BiotinACTH/anti-ACTH/APBA/SPCE1 and cortisol-AP/anticortisol/APBA/SPCE2 was much poorer retaining only approximately $30 \%$ of the initial value after twelve days from their preparation.

\subsection{Simultaneous Determination of ACTH and Cortisol in Certified Human Sera}

The usefulness of the dual immunosensor for the multiplexed analysis of real samples was evaluated by analyzing certified human serum samples. Calibration graphs for ACTH and cortisol were constructed from the reconstituted serum by appropriate dilution with the respective working buffer solutions in the 0.01 to $100 \mathrm{pg} / \mathrm{mL}$ ACTH and 0.1 to $200 \mathrm{ng} / \mathrm{mL}$ cortisol concentration ranges. It was observed that the calibration graphs constructed in diluted sera fitted perfectly with the calibration graphs obtained with standard solutions of the analytes depicted in Figure 6. Actually, the linear ranges of the calibration plots recorded with the serum samples exhibited slope values of $-0.507 \pm 0.006 \mu \mathrm{A}(r=0.999)$ for $\mathrm{ACTH}$, and $-1.6 \pm 0.1 \mu \mathrm{A} \quad(r=0.998)$ for cortisol, which were very similar to those obtained for ACTH and cortisol standard solutions $(-0.510 \mu \mathrm{A}$ and $-1.59 \mu \mathrm{A})$. These results, showing that no significant matrix effect existed, allowed the simultaneous determination of ACTH and cortisol to be performed by interpolation of the measured ip values into the calibration plots constructed with ACTH and cortisol standard solutions. The results obtained in the analyses carried out at four different concentration levels for each hormone in the samples are summarized in Table 2. As it can be seen, recoveries ranged between $98.3 \pm 0.1$ and $102 \pm 3 \%$ for ACTH and $99 \pm 9$ and $104 \pm 8 \%$ for cortisol. These results demonstrated fairly well the usefulness of the developed dual immunosensor for the multiplexed analysis of low ACTH and cortisol concentrations in human sera with practically no sample treatment.

\section{Conclusions}

In this paper, the development of an electrochemical multiple-analyte immunosensor for the ultrasensitive multiplexed determination of ACTH and cortisol hormones in 
Table 2. Simultaneous determination of ACTH and cortisol in certified human sera.

\begin{tabular}{llcccr}
\hline & Added & \multicolumn{3}{c}{ Found } \\
\hline ACTH, pg/mL & Cortisol $(\mathrm{ng} / \mathrm{mL})$ & ACTH $(\mathrm{pg} / \mathrm{mL})$ & Recovery $(\%)$ & Cortisol $(\mathrm{ng} / \mathrm{mL})$ & Recovery $(\%)$ \\
\hline 0.01 & 0.1 & $0.0102 \pm 0.0005$ & $99 \pm 5$ & $0.099 \pm 0.009$ & $99 \pm 9$ \\
0.1 & 2.5 & $0.102 \pm 0.003$ & $102 \pm 3$ & $2.6 \pm 0.2$ & $104 \pm 8$ \\
10 & 100 & $10.0 \pm 0.1$ & $100 \pm 1$ & $103 \pm 5$ & $103 \pm 5$ \\
100 & 200 & $98.3 \pm 0.1$ & $98.3 \pm 0.1$ & $204 \pm 7$ & $102 \pm 4$ \\
\hline
\end{tabular}

human serum is reported for the first time. The immunosensing approach involves the immobilization of antiACTH or anti-cortisol onto phenylboronic acid-modified dual screen printed carbon electrodes, competitive immunoassay implying biotinylated ACTH and alkaline phosphatase labelled streptavidin, or alkaline phosphatase labelled cortisol. and differential pulse voltammetric transduction of the affinity reactions. The developed dual immunosensor exhibits excellent analytical performance in terms of sensitivity, selectivity, wide range of quantifiable antigen concentrations, and inter-assay reproducibility. Therefore, this multiple-analyte immunosensor can be envisaged as a useful and affordable analytical tool for the rapid determination of $\mathrm{ACTH}$ and cortisol in clinical applications, as it has been outlined with the results obtained for the analysis of certified human serum samples.

\section{Acknowledgements}

Financial support from the Spanish Ministerio de Ciencia e Innovación (Projects DPS 2008-07005-C02-01, CTQ 2009-12650, CTQ 2009-09351) and Comunidad de Madrid S2009/PPQ-1642, programme AVANSENS is gratefully acknowledged.

\section{References}

[1] E. Davies, C. J. Keyon, R. Fraser, Steroids 1985, 45, 557.

[2] J. A. Norton, M. Li, J. Gillary, H. N. Le, Curr. Probl. Surg. 2001, 38, 487.

[3] E. R. Laws, Neurosurgery 1999, 45, 977.

[4] M. John, A. R. Lila, T. Bandgar, P. S. Menon, N. S. Shah, Pituitary 2010, 13, 48.

[5] G. Leibowitz, A. Tsur, S. D. Chayen, M. Salameh, I. Raz, E. Cerasi, D. J. Gross, Clin. Endocrinol. 1996, 44, 717.
[6] J. Newell-Price, P. Trainer, M. Besser, A. Grossman, Endocrine Rev. 1998, 19, 647.

[7] J. Brossaud, M. Bouton, B. Gatta, A. Tabarin, J.-B. Corcuff, Clin. Biochem. 2011, 44, 1160.

[8] M. S. Wilson, W. Y. Nie, Anal. Chem. 2006, 78, 6476.

[9] J. M. Fowler, D. K. Y. Wong, H. B. Halsall, W. R. Heineman, in Recent Developments in Electrochemical Immunoassays and Immunosensors (Eds: $\mathrm{X}$. Zhang, $\mathrm{H}$. Ju, J. Wang), Academic. Press, New York 2008, ch. 5, pp. 115-143.

[10] P. D’Orazio, Clin. Chim. Acta. 2011, 412, 1749.

[11] V. Serafín, M. Eguílaz, L. Agüí, P. Yáñez-Sedeño, J. M. Pingarrón, Electroanalysis 2011, 23, 169.

[12] M. Eguílaz, M. Moreno-Guzmán, S. Campuzano, A. González-Cortés, P. Yáñez-Sedeño, J. M. Pingarrón, Biosens. Bioelectron. 2010, 26, 517.

[13] M. Moreno-Guzmán, A. González-Cortés, P. Yáñez-Sedeño, J. M. Pingarrón, Anal. Chim. Acta 2011, 692, 125.

[14] M. Moreno-Guzmán, M. Eguílaz, S, Campuzano, A. González-Cortés, P. Yáñez-Sedeño, J. M. Pingarrón, Analyst 2010, 135, 1926.

[15] M. Moreno-Guzmán, I. Ojeda,, R. Villalonga, A. GonzálezCortés, P. Yáñez-Sedeño, J. M. Pingarrón, Biosens. Bioelectron. 2012, in press.

[16] J. A. Ho, W. A. Hsu, W. C. Liao, J. K. Chiu, M. L. Chen, H. C. Chang, C. C. Li, Biosens. Bioelectron. 2011, 26, 1021.

[17] P. Tijssen, in Laboratory Techniques in Biochemistry and Molecular Biology, Vol. 15 (Eds: R. H. Burdon, P. H. van Knippenberg), Elsevier, Amsterdam 1985, p. 414.

[18] G. Coneely, M. Aherne, H. Lu, G. G. Guilbault, Anal. Chim. Acta 2007, 583, 153.

[19] J. A. Talbot, J. W. Kane, A. White, Ann. Clin. Biochem. 2003, $40,453$.

[20] M. Vogeser, D. Engelhardt, K. Jacob, Clin. Chem. 2000, 46, 1998.

[21] S. Dobson, A. White, M. Hoadley, T. Lovgren, J. Ratcliffe, Clin. Chem. 1987, 33, 1747.

[22] L. Anfossi, C. Tozzi, C. Giovannoli, C. Baggiani, G. Giraudi, Anal. Chim. Acta 2002, 468, 315. 